Provided for non-commercial research and education use. Not for reproduction, distribution or commercial use.

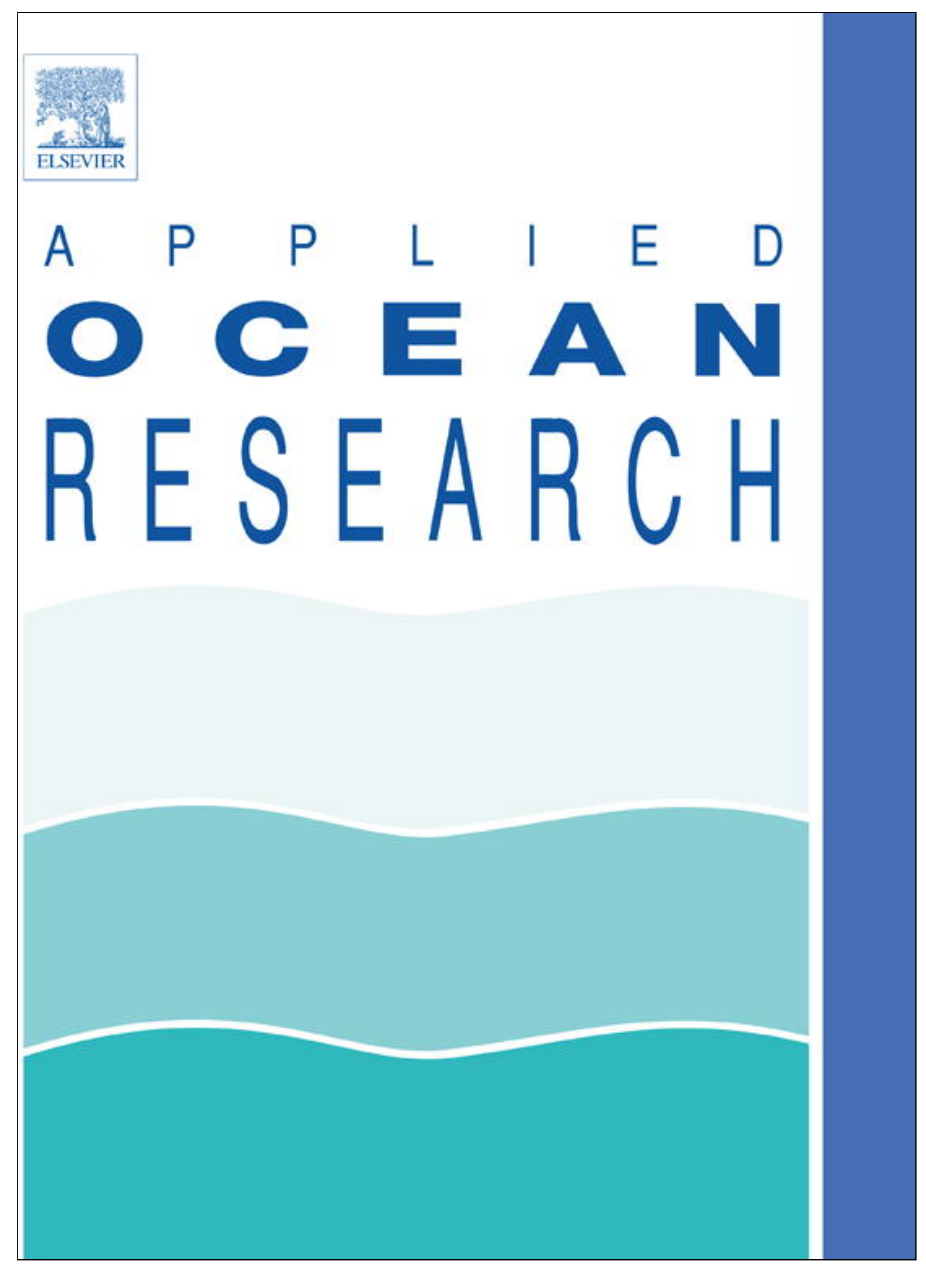

(This is a sample cover image for this issue. The actual cover is not yet available at this time.)

This article appeared in a journal published by Elsevier. The attached copy is furnished to the author for internal non-commercial research and education use, including for instruction at the authors institution and sharing with colleagues.

Other uses, including reproduction and distribution, or selling or licensing copies, or posting to personal, institutional or third party websites are prohibited.

In most cases authors are permitted to post their version of the article (e.g. in Word or Tex form) to their personal website or institutional repository. Authors requiring further information regarding Elsevier's archiving and manuscript policies are encouraged to visit:

http://www.elsevier.com/copyright 


\title{
Physical modeling and swirling strength analysis of vortex shedding from near-bed piggyback pipelines
}

\author{
Zhi-Peng Zang, Fu-Ping Gao*, Jin-Sheng Cui \\ Institute of Mechanics, Chinese Academy of Sciences, Beijing 100190, China
}

\section{A R T I C L E I N F O}

Article history:

Received 9 August 2012

Received in revised form 30 December 2012

Accepted 1 January 2013

\section{Keywords:}

Piggyback pipelines

Vortex shedding

Swirling strength

VIV Suppression

\begin{abstract}
A B S T R A C T
The vortex shedding from near-bed piggyback pipelines in a steady flow has been investigated experimentally in a large water flume. A specially arranged PIV system with upward-illumination of pulsed laser arrays from the flume bottom was employed for the flow visualization and quantitative measurement of the lee-wake flow in a sub-critical regime around the piggyback pipelines in the proximity of a plane boundary. Based on dimensional analyses, a dimensionless maximum swirling strength $\left(W_{\mathrm{m}}\right)$ is used for analyzing the vortex shedding intensity and its frequency. Time-averaged swirling strength analyses indicate that the lee-wake patterns for the near-bed piggyback pipelines are dependent on the configuration factors, including the gapto-diameter ratio $(e / D)$, the spacing-to-diameter ratio $(G / D)$, and the diameter ratio of two pipes $(d / D)$, etc. The swirling strength in the lee-wake is obviously asymmetric for piggyback pipelines with bed proximity. For the fixed values of $G / D$ and $d / D$, the maximum swirling strength decreases with the decrease of $e / D$. Moreover, for the examined $G / D$ range $(0 \leq G / D \leq 0.5)$, minimum values of $W_{\mathrm{m}}$ and corresponding VIV amplitude for the piggyback pipelines are evidently within the same range of spacing-to-diameter ratio $G / D$ $\approx 0.05-0.20$.
\end{abstract}

Crown Copyright (c) 2013 Published by Elsevier Ltd. All rights reserved.

\section{Introduction}

With the rapid development of offshore oil and gas engineering, piggyback pipelines have been increasingly used for technical and economic reasons. The piggyback pipelines generally comprise a primary flowline for exporting the hydrocarbon flow away from the field and a secondary flowline carrying water, injection chemicals, etc. to the field. Compared with a single pipeline, the flow around piggyback pipelines is more complicated due to the existence of the piggyback. The vortex shedding from the main pipe may be suppressed by both the piggyback and the sea bottom in some cases, bringing alleviation of vortex-induced vibrations (VIVs). To intensively investigate vortex shedding characteristics of piggyback pipelines and the optimized configurations for vortex shedding suppression would be beneficial to the design and operation of piggyback pipelines.

In the past decades, the near-wall effects on vortex shedding and hydrodynamic loading of a single pipeline have been studied experimentally or numerically by many researchers. Bearman and Zdravkovich [1] analyzed the spectral of hot-wire signals in the near wake of the pipe to determine the strength and frequency of the vortex shedding. It was shown that vortex shedding is suppressed when gap ratio $e / D$ is smaller than 0.3 . Grass et al. [2] indicated that the critical gap ratio $(e / D)_{\mathrm{cr}}$ for vortex shedding suppression varies in the

\footnotetext{
* Corresponding author. Tel.: +8610 82544189; fax: +861062561284.

E-mail address: fpgao@imech.ac.cn (F.-P. Gao).
}

range of 0.3-0.5 dependent on the boundary layer thickness, Lei et al. [3] observed that there are three different flow regimes for the near-wall pipe by examining the root-mean-square values of the lift force. The particle image velocimetry (PIV) technique was employed recently by Price et al. [4], Wang and Tan [5] and Lin et al. [6] to measure the wake flow fields around a single circular cylinder near a plane boundary. The lee-wake patterns and the characteristics of vortex shedding from the cylinder have been examined quantitatively.

Significant studies on piggyback pipelines have been conducted only in the past two decades. Li and Zhang [7] established relationships between hydrodynamic force coefficients and $K C$ number for piggyback pipelines under combined waves and currents. An equivalent-diameter method was also proposed in their study. Chung and Conti [8] conducted a series of tests on hydrodynamic forces and wake flow patterns for a variety of pipe configurations. Their tests indicated that a pipe enlaced with power cables or with a perforated shroud reduced effectively the vortex shedding intensity and hydrodynamic forces compared with a bare pipe. Kalahatgi and Sayer [9] found that the existence of a piggyback increased the drag force on piggyback pipelines by $50-100 \%$ compared with that for a single pipeline at $R e$ ranging from $9.0 \times 10^{4}$ to $3.0 \times 10^{5}$. The direction of the transverse lift force was dependent on $R e$ and $e / D$. Williamson [10] found that there were pronounced increases in hydrodynamic forces and the vortex shedding frequency for a small pipe approaching a larger one in oscillatory flows. Zeitoun et al. [11] and Brankovic et al. [12] showed that the piggyback increases the drag force and decreases the lift force on piggyback pipelines compared with a single 
pipe. The equivalent diameter method widely adopted in engineering designs may be conservative in predicting the lateral displacement.

Besides the aforementioned experimental studies, numerical methods have also been used to study the flow behaviors around piggyback pipelines in complicated marine environments. Kamarudin et al. $[13,14]$ numerically studied the hydrodynamic forces on a pipeline bundle with a variety of orientations of the piggyback. The main pipe with an upstream or downstream piggyback experienced smaller mean drag force than other cases, while the maximum lift force on the main pipe occurred with a $\pi / 4$ arrangement of the piggyback. Their results also showed that the equivalent-diameter method may underestimate the forces on the pipeline bundle. Zhao et al. $[15,16]$ developed a numerical model for simulating the flows around two pipes with different diameters at low $R e$ and high $R e$, respectively. In Zhao et al. [17] the response of piggyback pipelines near a plane seabed in a steady current at $R e=2 \times 10^{4}$ was further examined intensively with the effects of the gap between the main pipe and the seabed surface, and the spacing between two pipes, etc.

Most of the existing studies on the response of piggyback pipelines focused on the amplitude and frequency of hydrodynamic forces. However, the lee-wake flow field and vortex shedding from the nearwall piggyback pipelines have not been measured quantitatively and analyzed intensively yet. In the previous experimental observations the single-point signal monitoring (hot-wire [1], LDV [18], etc.) and flow visualization techniques (smoke-line [1], hydrogen bubble [19], dye injection $[8,20]$, etc.) had been employed for the measurement of the wake flow around structures. The lee wake patterns were observed qualitatively with those flow visualization techniques.

The objectives of this work are to quantitatively measure the leewake vortex shedding from near-wall piggyback pipelines, and to further examine the effect of configuration factors on vortex shedding. To this aim a specially arranged PIV system with upward-illumination of pulsed laser arrays from the flume bottom was employed for the flow visualization and quantitative measurement of the lee-wake flow in a sub-critical regime around the piggyback pipelines in the proximity of a plane boundary. The detailed information in the 2D lee-wake flow at the near-wall piggyback pipelines could be obtained, including velocity vectors, vorticity, swirling strength, Reynolds stress, etc. Moreover, a non-dimensional swirling strength $(W)$ is proposed and used to analyze the vortex shedding intensity and its frequency. Piggyback pipelines with the secondary pipe installed above the main pipe are taken into account, as this configuration is most popular in practical engineering. The structural factors for the examined configuration of piggyback pipelines include the gap-to-diameter ratio $(e / D)$, the spacing-to-diameter ratio $(G / D)$, and the diameter ratio of two pipes $(d / D)$, etc. Based on the dimensional analyses for swirling strength, the optimized configurations for the suppression of vortex shedding are proposed, which is further compared with the corresponding VIV response of the piggyback pipelines.

\section{Experimental setup and physical modeling method}

The flow measurements were carried out in a water flume $(52.0 \mathrm{~m}$ length, $1.0 \mathrm{~m}$ width, and $1.5 \mathrm{~m}$ depth), at the Institute of Mechanics, Chinese Academy of Sciences. A specially arranged PIV measurement was employed as shown in Fig. 1(a). At the test section, the ground of flume is replaced with a piece of $1.0 \mathrm{~m} \times 1.0 \mathrm{~m}$ transparent glass installed $16.0 \mathrm{~m}$ downstream of the flow inlet. Through the transparent glass, double-pulsed laser sheets generated by a LaVision PIV system illuminate the flow field around the piggyback pipelines from the bottom side. This experimental arrangement can capture the flow details between the pipe and the bottom wall well, and effectively reduce the reflection of laser light by the water surface. The water depth during tests is kept at $0.5 \mathrm{~m}$. The model of the main pipe is made of a plexiglass tube with an outer diameter of $D=80 \mathrm{~mm}$ and with a gap to the bottom $e$. The piggyback is made of a plexiglass rod with a diameter of $d=20 \mathrm{~mm}$ installed above the main pipe with spacing $G$. The length of the pipeline model is $L=980 \mathrm{~mm}$. The aspect ratio of $L / D$ is considered large enough to ensure a $2 D$ flow in the central region of the pipe, avoiding the boundary effect of sidewalls. The configuration of piggyback pipelines and the coordinate system is shown in Fig. 1(b).

In the PIV image processing the multi-pass with decreasing window size technique was applied to improve the resolution of vector fields and produce less erroneous vectors. The iteration started with an initial interrogation window size of $64 \times 64$ pixels, and then stepped down to the window size of $32 \times 32$ pixels with an overlapping of 50\%. This interrogation and evaluation procedure yielded approximately 5600 velocity vectors $(u, v)$ for each image pair. Based on a compromise between the requirements of recording a large field of view and resolving detailed flow structures, the PIV viewing area for most cases was chosen at $320 \mathrm{~mm} \times 240 \mathrm{~mm}$ (i.e. $4.0 \times 3.0$ $D$ ) in the $x-y$ plane. Therefore, the spatial resolution for this setup is about $3.6 \times 3.6 \mathrm{~mm}$ (i.e. $0.045 \times 0.045 \mathrm{D}$ ). For each test case, 300 flow samples were acquired at the frequency of $5.03 \mathrm{~Hz}$ (i.e. $59.64 \mathrm{~s}$ recordings). Following the procedure for the accuracy analysis, due to the limitation of the number of PIV samples [21], the standard deviation of velocity components $u$ and $v$ in this study was estimated to be $5 \%$. The time interval between successive flow fields $\Delta t$ may be expressed as a fraction of the vortex shedding period $T_{\mathrm{s}}$, determined from the vortex-shedding frequency, where $\Delta t / T_{S}=\Delta t u / D \cdot S t$ (St, the Strouhal number, $\approx 0.20$ ). In this study, the time difference between the successive pairs of flow fields was $\Delta t \approx 0.2 \mathrm{~s}$, corresponding to $0.075-0.15 T_{\mathrm{s}}$. Sufficient information can be acquired to represent the fluid behavior of vortex shedding over the recording period. The free stream velocity $3.0 \mathrm{~m}$ upstream of the piggyback pipeline model was measured using acoustic Doppler velocimetry (ADV). For the laminar boundary layer on a plate, the boundary layer thickness can be estimated at the point where the velocity $u$ reaches $99 \%$ of the free stream velocity $U$ [22]. The boundary layer thickness in the examined cases is estimated as ranging from $2.8 \mathrm{~cm}$ to $5.2 \mathrm{~cm}$, which is corresponding to $0.35-0.65 D$.

In the past decades various vortex identification methods including closed or spiral pathlines [23], minimum local pressure [24], vorticity magnitude $[25,26]$, etc., have been used to interpret the vortical structures in an instantaneous flow field. However, each criterion has its own limitation and may fail in certain situations. For vorticity magnitude, which is widely used in qualifying the intensity of vortices, it may fail to identify vortical motions in a boundary layer flow [27]. This is because the shear motion can also generate high magnitude of vorticity. There apparently exist boundary layer flows around the near-bed piggyback pipelines. Hence, in this study the magnitude of swirling strength [28], which had been proven to discriminate between vortices and shear motions successfully, is applied to qualify the intensity of vortex shedding from the piggyback pipelines. The definition of swirling strength is related to the discriminant of complex eigenvalues of the local velocity gradient tensor. For a 2D flow, the velocity gradient tensor can be expressed as:

$\nabla \vec{u}=\left[\begin{array}{ll}\frac{\partial u}{\partial x} & \frac{\partial u}{\partial y} \\ \frac{\partial v}{\partial x} & \frac{\partial v}{\partial y}\end{array}\right]$

The discriminant of the characteristic equation of the velocity gradient tensor is [27,29]:

$\Delta=\left(\frac{\partial u}{\partial x}+\frac{\partial v}{\partial y}\right)^{2}-4\left(\frac{\partial u}{\partial x} \frac{\partial v}{\partial y}-\frac{\partial u}{\partial y} \frac{\partial v}{\partial x}\right)$

$\nabla \vec{u}$ will either have two real eigenvalues $(\Delta \geq 0)$ or a pair of complex conjugate eigenvalues $(\Delta<0)$ dependent on the value of $\Delta$. According to Chong et al. [28], the water particle would exhibit a swirling motion only when $\nabla \vec{u}$ has a pair of complex eigenvaues $\lambda_{r} \pm \lambda_{i}$, i.e. $\Delta<$ 


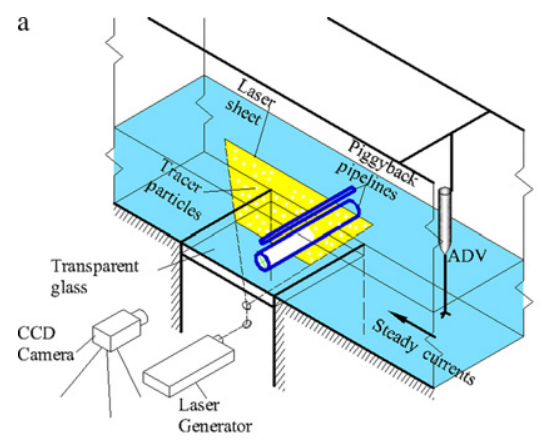

Fig. 1. (a) Experimental setup for PIV measurements and (b) configuration of piggyback pipelines.

0. $\lambda_{i}^{-1}$ represents the period required for a water particle swirling once around the vortex core. Thus, for a pure shear flow, the water particle swirls in an infinitely long elliptical orbit and the period of the swirling motion tends to be infinite, corresponding $\lambda_{i}=0$; while $\lambda_{i}>0$ corresponds to a shorter and more circular swirling motion, i.e. vortex. Zhou et al. [30] have also shown that the strength of any local swirling motion can be quantified by the complex part of the eigenvalues.

Here, the swirling strength is defined as [31]:

$\Omega=\max \left(0, \frac{-\Delta}{4}\right)$

The unit of $\Omega$ is $\mathrm{s}^{-2}$. The value of $\Omega$ is proportional to the square of the frequency for a water particle swirling once. It is a function of flow and structure variables:

$\Omega=F(U, v, D, d, e, G, \ldots)$

Then, a non-dimensional form of swirling strength is proposed based on the vortex shedding frequency of the main pipe:

$W=\left(\frac{f_{w}}{f_{S}}\right)^{2}=\frac{D^{2} \Omega}{(S t \cdot U)^{2}}$

where $W$ is the non-dimensional swirling strength, $f_{W}$ is the frequency for water particles swirling, $f_{s}$ is the vortex shedding frequency of the main pipe, $S t$ is the Strouhal number. Following a dimensional analysis, $W$ can be expressed as a function:

$W=F^{\prime}\left(R e, \frac{e}{D}, \frac{G}{D}, \frac{d}{D}, \ldots\right)$

where the Reynolds number is defined based on the diameter of the main pipe, namely, $R e=U D / v, v$ is the kinematic viscosity of the water. The effect of the configuration factors including $e / D, G / D$ and $d / D$ on $W$ will be discussed in following sections.

\section{Results and discussion: swirling strength analysis}

\subsection{Swirling strength of the flow field at the rear of a single pipeline} $(G / D \approx \infty)$

The single pipeline can be considered as an extreme configuration of piggyback pipelines with the spacing between two pipes being infinitely large, i.e. $G / D \approx \infty$. In this section the wake flow fields around a single pipeline were measured and analyzed in terms of $W$ to validate the effectiveness of the swirling strength method in qualifying the vortex shedding intensity. As the time-average values of the characteristic variables are often used by researchers for studying a fluctuating flow field, the vortex shedding intensity of piggyback pipelines is quantified with the peak value of time-averaged fields of the non-dimensional swirling strength over the recording period, denoted as $W_{\mathrm{m}}$. The vortex shedding frequency is acquired with FFT analysis of the instantaneous non-dimensional swirling strength.

The flow measurements for a wall-free single pipe $(e / D=1.0)$ were conducted first. Fig. 2(a) shows the mean streamlines in the nearwake at $R e=1.5 \times 10^{4}$ and Fig. 2(b) is the corresponding contour plot of the non-dimensional swirling strength. There are two recirculation regions, i.e. separation-reattachment zones, downstream of the pipe approximately symmetric about the centerline of the pipe. It can be seen that the swirling strength field can well reflect the vortex regions corresponding to the streamlines. The peak values of the swirling strength field, $W_{\mathrm{m}}$, are located at the center of vortex regions (approximately at $x / D=1.0$, in Fig. $2(\mathrm{~b})$ ). The recirculation length $L_{\mathrm{S}}$ is defined from the downstream edge of the pipe to the location where the mean magnitude of $u$ reaches zero, as shown in Fig. $2(a)$. The variation of the non-dimensional recirculation length with Re number is shown in Fig. 3 with Re ranging from $2.5 \times 10^{3}$ to 4.0 $\times 10^{4}$. The measured values of $L_{S} / D$ published by Norberg [18], Ma et al. [32] and Govardhan and Williamson [33] are also plotted in Fig. 3 for comparison. $L_{S} / D$ is almost kept at a value of 1.5 within the examined Re range. The value of $L_{S} / D$ indicates the range for measuring fluctuations of $W$ around a pipeline.

The variation of the peak values of the mean swirling strength, $W_{\mathrm{m}}$, for a single pipe with Re number is plotted in Fig. 4. The value of $W_{\mathrm{m}}$ is almost constant with $R e$ in the examined (sub-critical) flow regime. This phenomenon is also similar to that for the non-dimensional vortex shedding frequency $(S t)$ independent of Re in the sub-critical regime.

The near-wall effect on the vortex shedding from a single pipeline was obtained in terms of the non-dimensional swirling strength. Fig. 5 shows the variation of $W_{\mathrm{m}}$ with $e / D$ at two Re numbers of $1.5 \times$ $10^{4}$ and $3.0 \times 10^{4} . W_{\mathrm{m}}$ decreases with the decrease of $e / D$ when $e / D$ $<0.4$, owing to the suppression of vortices shedding from the pipe by the bottom wall. For $e / D \geq 0.4, W_{\mathrm{m}}$ is almost kept at a constant value, which means that the vortices are shedding freely from the pipe with no inhibition. The critical gap ratio, $(e / D)_{\mathrm{cr}}$, for suppression of vortex shedding by the bottom is around 0.4 , which is very close to the values published in most literatures, such as, 0.3 by Bearman and Zdravkovich [1], 0.3-0.5 by Grass et al. [2] and 0.5 by Price et al. [4]. The slight difference among these values of $(e / D)_{\mathrm{cr}}$ stems mainly from the different boundary layer thickness.

From the above analyses it is reasonable to assume that the intensity of vortices shedding can be represented quantitatively by the non-dimensional swirling strength. In the following work the vortex shedding characteristics of piggyback pipelines in a steady current will be investigated based on the non-dimensional swirling strength. 


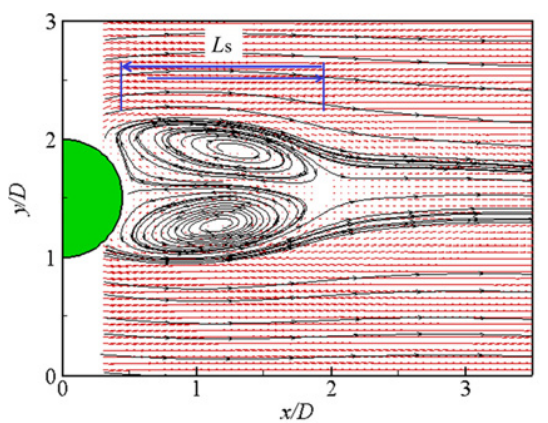

(a)

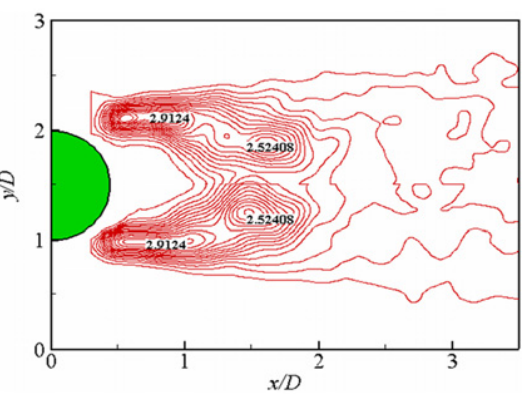

(b)

Fig. 2. Time-averaged flow fields at the rear of a single pipe: (a) streamlines and (b) non-dimensional swirling strength $\left(G / D \approx \infty, e / D=1.0\right.$, and $\left.R e=1.5 \times 10^{4}\right)$.

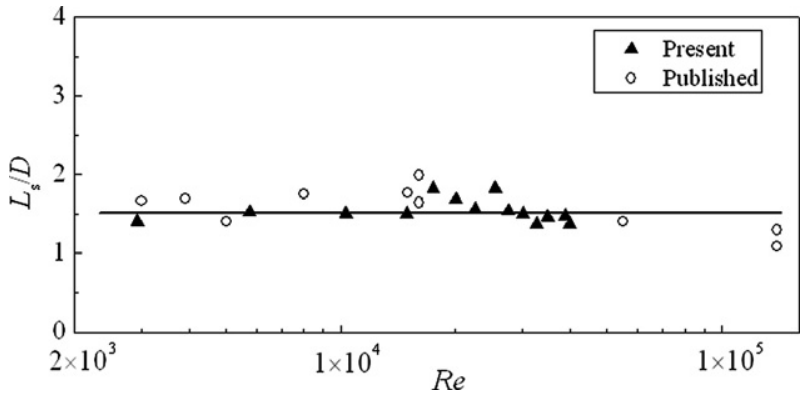

Fig. 3. Variation of the recirculation length $L_{S} / D$ with $R e$ for a single pipeline $(G / D \approx$ $\infty$ and $e / D=1.0)$.

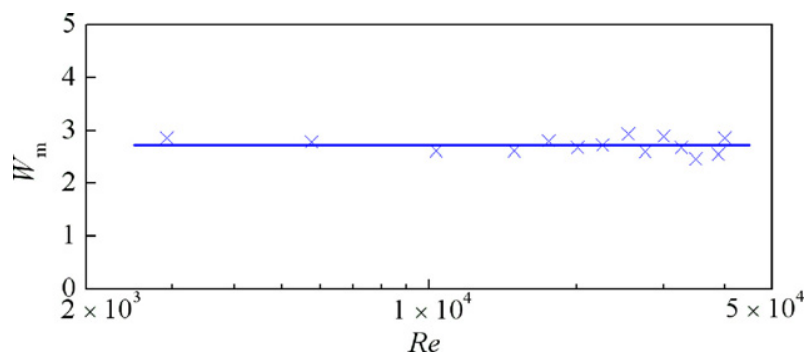

Fig. 4. Variation of the maximum non-dimensional swirling strength $W_{\mathrm{m}}$ with $R e$ for a single pipeline $(G / D \approx \infty$ and $e / D=1.0)$

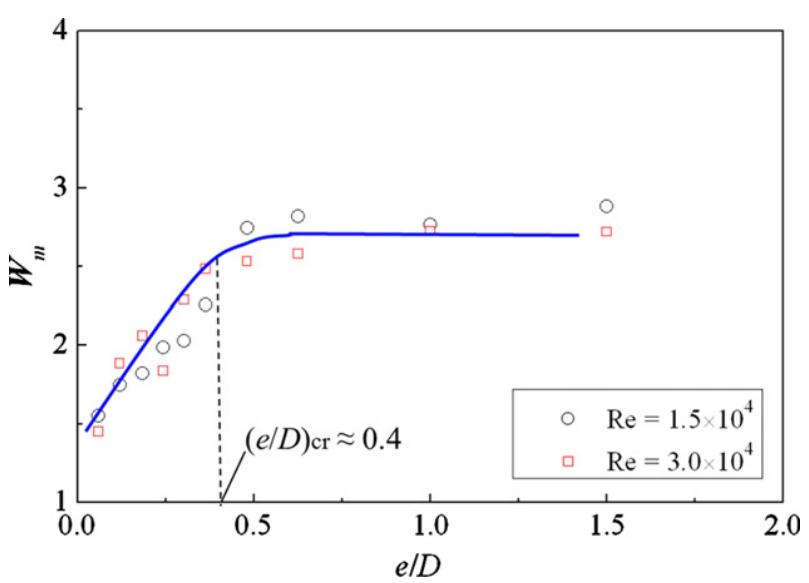

Fig. 5. Variation of the maximum non-dimensional swirling strength $W_{\mathrm{m}}$ with $e / D$ for a single pipeline $(G / D \approx \infty$ and $e / D=1.0)$.

\subsection{Swirling strength of the flow field at the rear of piggyback pipelines}

\subsubsection{Effect of $e / D$ and $G / D$ on wake flow patterns}

The wake flows around piggyback pipelines were measured at $R e$ $=3.0 \times 10^{4}$ with a small value of diameter ratio of $d / D=0.25$. The gap-to-diameter ratio for the main pipe, $e / D$, ranges from 0 to 1.0. The spacing-to-diameter for two pipes, $G / D$, ranges in 0.0-0.5.

Fig. 6 shows the contour plots of the time-averaged nondimensional swirling strength around piggyback pipelines under a bottom-free condition $(e / D=1.0)$. In Fig. $6(\mathrm{a})$, the piggyback contacts directly above the main pipe $(G / D=0)$. The vortex regions behind the piggyback pipelines are approximately symmetric about the centerline of the piggyback pipeline as that for a single pipe in Fig. 2(b). The shear layers separated freely from both sides of the equivalent large pipe without any disturbance. Regular vortex shedding is formed behind the piggyback pipelines, and contacting each other in the near wake. In Fig. 6(b), there is a small spacing between the two pipes $(G / D=0.125)$. The vortex regions behind the piggyback pipelines are not as symmetric as that for $G / D=0$. It seems that there are two or three small vortex cores near the upper side of the piggyback pipeline forming a large vortex region due to the vortices shed from the piggyback interacting with that from the main pipe. With the increase of the spacing the interference between the two pipes is weakened gradually. When $G / D$ is at a large value (e.g. Fig. $6(\mathrm{c})$ and (d)), the two pipes are free from each other, i.e. they behave as two separate single pipes. There is regular vortex shedding from each pipe with no interference and suppression.

Fig. 7 shows the contour plots of the time-averaged nondimensional swirling strength around piggyback pipelines near the bottom $(e / D=0.125)$. The corresponding plot for a single pipe is also shown for comparisons. Under this condition there are no symmetric vortex regions behind the pipeline as the vortex shedding from the lower side of main pipe is suppressed by the bottom. The vortex shedding intensity at the lower side is much smaller than that at the upper side. In Fig. 7(a) and (d), it can be seen that the wake flow patterns for these two configurations are very similar to each other because the piggyback pipelines with two adjacent pipes $(G / D=0)$ behave as a single pipeline $(G / D \approx \infty)$. For a medium value of $G / D(=0.125$, in Fig. 7(b)), there are no clear vortex regions near the upper side of the piggyback pipelines due to the interactions between the vortex shedding from the main pipe and that from the piggyback. For a relatively large value of $G / D$, the vortex shedding from both the main pipe and the piggyback are regular with no interactions with each other. There are three distinct vortex regions near the upper side of the piggyback pipeline as shown in Fig. 6(c).

Fig. 8 shows a series of contour plots of the time-averaged nondimensional swirling strength around pipelines mounted on the bottom $(e / D=0)$ with and without a piggyback. As the main pipe is mounted on the bottom the vortex shedding at the lower side of the pipe was suppressed entirely. The difference between the wake flow 


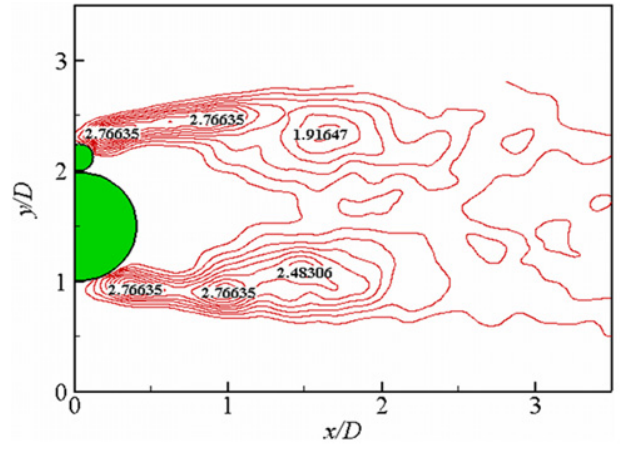

(a)

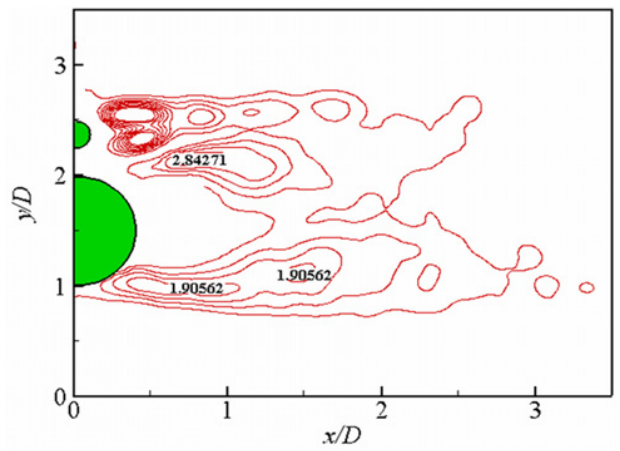

(c)

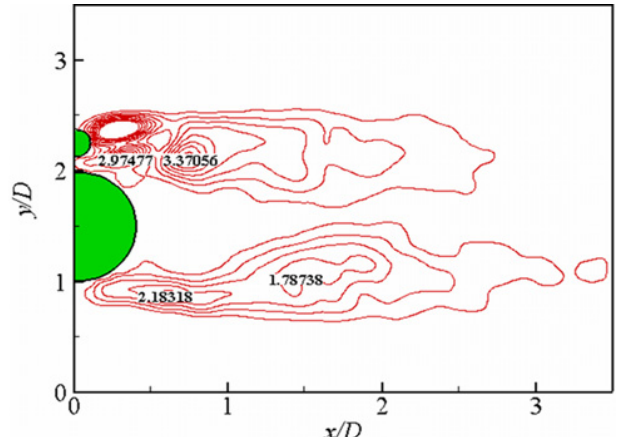

(b)

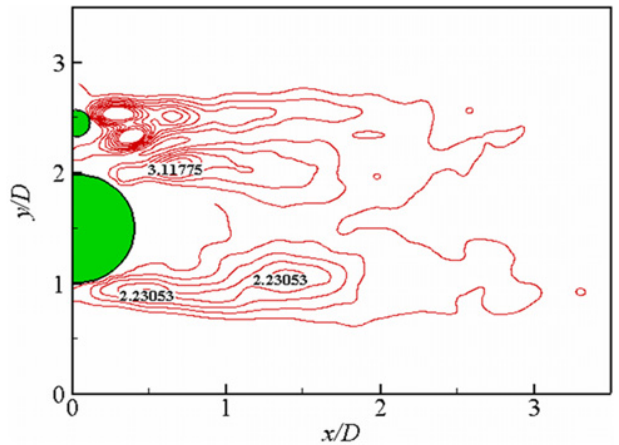

(d)

Fig. 6. Time-averaged fields of the non-dimensional swirling strength for bottom-free piggyback pipelines: (a) $G / D=0$, (b) $G / D=0.125$, (c) $G / D=0.25$, and (d) $G / D=0.325$ ( $e / D$ $=1.0, d / D=0.25$, and $R e=3.0 \times 10^{4}$ ).

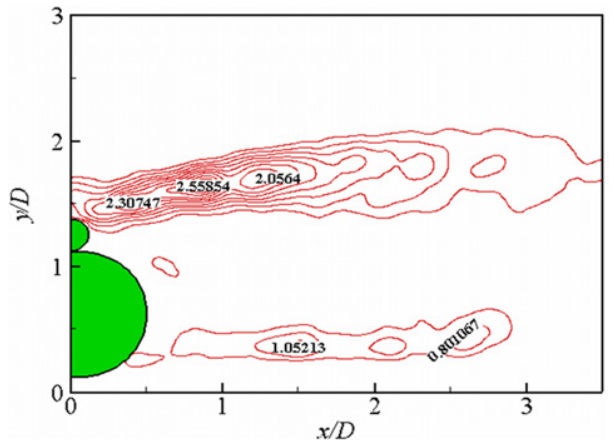

(a)

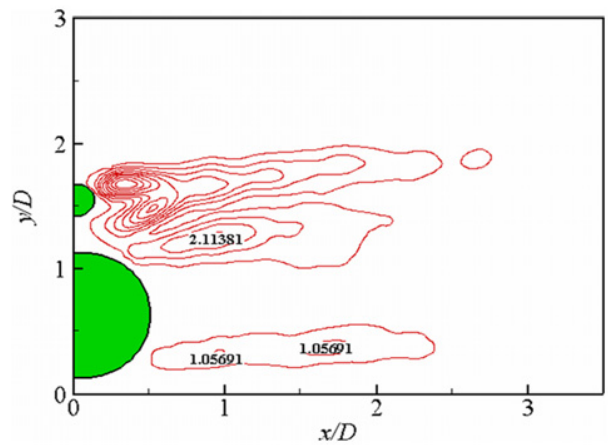

(c)

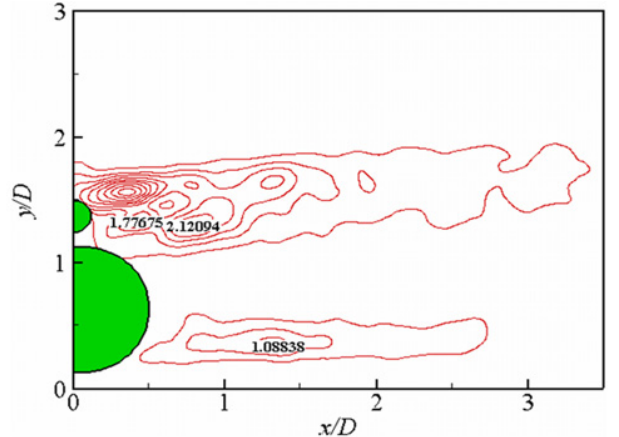

(b)

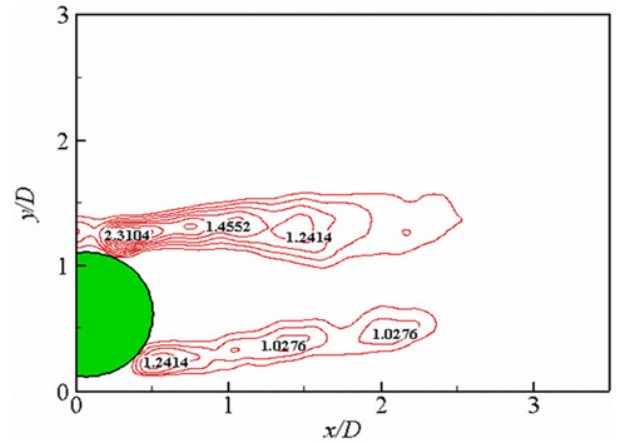

(d)

Fig. 7. Time-averaged fields of the non-dimensional swirling strength for near-bottom piggyback pipelines: $e / D=0.125, d / D=0.25$, and $R e=3.0 \times 10^{4}$. (a) $G / D=0$, (b) $G / D=$ 0.125 , (c) $G / D=0.30$, and (d) $G / D \approx \infty$ (single pipeline). 
patterns lies in the upper wake regions. In Fig. 8(a) and (d), for $G / D=$ 0 and $G / D \approx \infty$, the two vortex shedding modes are similar to each other. The shear layers separate consecutively from the upper side of piggyback pipelines with one-side vortex shedding. There is only a narrow vortex region at the upper side in the streamline direction. In Fig. 8 (b), for a medium spacing $(G / D=0.125)$, the shear layers separate from both sides of the piggyback but not regularly, owing to the interference by the main pipe. The wake flows behind the two pipes interact with each other and the vortices shed from the two pipes merge into one vortex region in the far wake. In Fig. 8(c), for a large spacing $(G / D=0.3)$, the piggyback behaves as a wall-free pipe. There is regular vortex shedding from the piggyback. The shear layers separation from the upper side of the main pipe is also in a stable manner. There are three distinct vortex regions side by side near the upper side of piggyback pipelines.

From the above contour plots of the non-dimensional swirling strength around piggyback pipelines it can be seen that the wake flow patterns around the piggyback pipelines are very dependent on $e / D$ and $G / D$. In Fig. 8(a), for small $e / D$ and $G / D$ there is no vortex shedding from the lower side of the piggyback pipeline. The two pipes are so close that they behave as a larger pipe with an equivalent diameter. Under this configuration the periodic vortex shedding was entirely suppressed by the bottom wall. There is only one vortex region formed near the upper side of the piggyback pipelines.

For a large value of $e / D$ and small value of $G / D$ the two pipes are close enough to each other and the whole body is free from the bottom wall. The vortices shed alternatingly from both upper and lower sides of the piggyback pipeline as that from a wall-free single pipe. In the non-dimensional swirling strength field there are two vortex regions formed on both sides of the piggyback pipelines, as shown in Fig. 6(a).

For a small value of $e / D$ and large value of $G / D$ the two pipes are free of each other and the whole structure is close to the bottom wall. The vortex shed from the main pipe is suppressed by the bottom and there is only a large vortex region near the upper side of the main pipe. As the piggyback is free from the main pipe the vortices shed regularly from the piggyback. There are three vortex regions around the piggyback pipelines, as shown in Fig. 8(c).

For a large value of $e / D$ and $G / D$, the two pipes and the bottom wall are free from each other. Regular vortex shedding is formed from both the main pipe and the piggyback. There are two vortex regions behind each pipe. Therefore, four vortex regions are found behind the piggyback pipelines as shown in Fig. 6(c).

\subsubsection{Effect of $d / D$ on vortex shedding from piggyback pipelines}

A study on the effect of diameter ratio $d / D$ on vortex shedding characteristics of piggyback pipelines is conducted further. The piggybacks with diameter of $20 \mathrm{~mm}, 30 \mathrm{~mm}$ and $40 \mathrm{~mm}$ are taken into account, with the corresponding diameter ratios of $d / D=0.25,0.375$ and 0.5 , respectively.

Fig. 9 shows the contour plots of the time-averaged nondimensional swirling strength around piggyback pipelines for various $G / D$ with a large diameter ratio $(d / D=0.5)$. Generally, the flow characteristics for piggyback pipelines with a large value of $d / D$ are similar to that for a small $d / D$ configuration, but the vortex regions are more turbulent. For $G / D=0$ (Fig. 9(a)), the two pipes behave as a single body. Owing to the large diameter ratio the wake flow patterns behind the piggyback pipelines are not as symmetric as that for a small diameter ratio configuration (Fig. 6(a)). The influence of piggyback on the main pipe decreases with the increase of $G / D$. The wake flow patterns behind the piggyback pipelines also show different numbers of vortex regions depending on the value of $G / D$. For the large $d / D$ configuration a larger spacing ratio between two pipes (i.e. $G / D \geq 0.5)$ is required than that for the small diameter ratio configuration (i.e. $G / D \geq 0.3$ ) so that the piggyback and the main pipe behave as two separate pipes, i.e. with two vortex regions behind each pipe.

The intensity of vortex shedding from the main pipe at three values of $d / D$ was quantified with the maximum swirling strength $\left(W_{\mathrm{m}}\right)$ as shown in Fig. 10. $W_{\mathrm{m}}$ decreases with the increase of $d / D$ for a constant value of $G / D$, especially for a small value of $G / D$. That means the vortex shedding from the main pipe was suppressed by the piggyback more significantly at a larger value of $d / D$. This can be easily understood by comparing the results for the two limit values of $d / D=0$ and infinity. For $d / D=0$ there is no piggyback above the main pipe. The vortex shedding occurs freely with no inhibition. For $d / D \approx \infty$ the piggyback can be considered as large as a plane wall. The vortex shedding is suppressed most significantly in this case.

\subsection{Optimal configurations of piggyback pipelines}

From the above discussions it is can be seen that the vortex shedding characteristics of piggyback pipelines are very dependent on $e$ / $D$ and $G / D$. As the vortex shedding is the main cause of dynamic responses of pipelines in steady currents the optimal configuration of piggyback pipelines will be obtained from aspects of both the vortex shedding suppression and the minimum amplitude of VIV.

\subsubsection{Optimal configurations based on vortex shedding suppression}

(1) Vortex shedding intensity. The vortex shedding intensity for piggyback pipelines discussed in Section 3.2.1 is studied quantitatively here. As the diameter ratio of two pipes is small $(d / D=0.25)$ in these cases the vortex shedding from the main pipe dominates the dynamic responses of the piggyback pipeline. The value of $W_{\mathrm{m}}$ around the main pipe is plotted with the variation of $e / D$ and $G / D$ in Fig. 11. The value of $W_{\mathrm{m}}$ for a wall-free single pipe $(G / D \approx \infty, e / D=1.0)$ is also plotted as reference.

Generally, $W_{\mathrm{m}}$ for the main pipe decreases with the decrease of $e / D$ when $G / D$ is kept at a certain constant value. That is to say, the vortex shedding from piggyback pipelines is suppressed by the bottom wall when $e / D$ is a small value. For a constant value of $e / D, W_{\mathrm{m}}$ decreases with the increase of $G / D$ when $G / D$ is small, then increases with the increase of $G / D$ and reaches a constant value thereafter.

For wall-free piggyback pipelines (i.e. $e / D=1.0$ ), when $G / D=0$, the two pipes contact each other and behave as a larger single pipe. Regular vortex shedding is formed behind the piggyback pipelines and the value of $W_{\mathrm{m}}$ is also larger than that for a single pipe (i.e. $G / D$ $\approx \infty)$. When two pipes are at a medium distance the wake flows of the two pipes interfere with each other. Then, the vortex shedding from the main pipe is disturbed by the piggyback. The value of $W_{\mathrm{m}}$ is smaller than that for a single pipe. For a large value of $G / D(>0.3)$, the piggyback is far from the main pipe. The piggyback pipelines behave as two separate pipes. Therefore, the vortices are shedding freely from two separate pipes without interference with each other, and $W_{\mathrm{m}}$ reaches the value for a single pipe again. For other values of $e / D$, $W_{\mathrm{m}}$ also has the same trend as that for $e / D=1.0$.

In Fig. 11, the dashed line indicates the value of $G / D$ for the smallest value of $W_{\mathrm{m}}$ with the various $e / D$. The value of $G / D$ for the corresponding smallest value of $W_{\mathrm{m}}$ increases with the increase of $e / D$ and convergences at $\approx 0.20$ for a large value of $e / D$. The proper range of $G / D$ for the smallest vortex shedding intensity is in $0.05-0.20$ in the examined cases.

(2) Vortex shedding frequency. The fluctuations of the nondimensional swirling strength near the upper side of the main pipe (point A in Fig. 1(b)) were monitored and analyzed with FFT to study the vortex shedding frequency for the main pipe. Fig. 12 shows the variation of the normalized vortex shedding frequency, St, with various value of $e / D$ and $G / D$ at $R e=3.0 \times 10^{4}$. According to Sumer and Fredsøe [34], St number increases when the vortex shedding from a structure is suppressed. In Fig. 12, St increases with the increase of $e / D$ when $G / D$ is set at a certain constant value due to the suppression of vortex shedding by the bottom wall. For a constant value of $e / D$ 


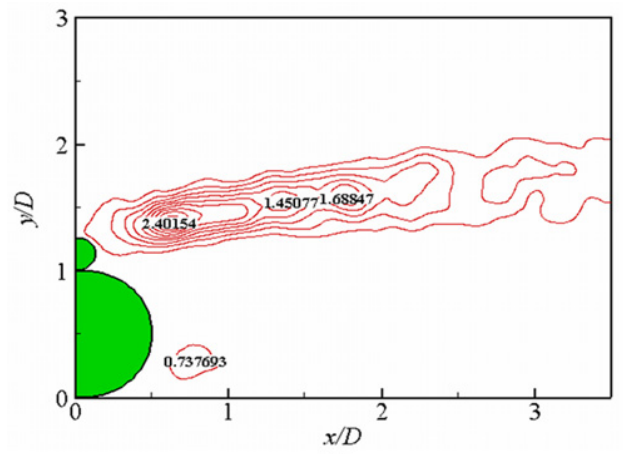

(a)

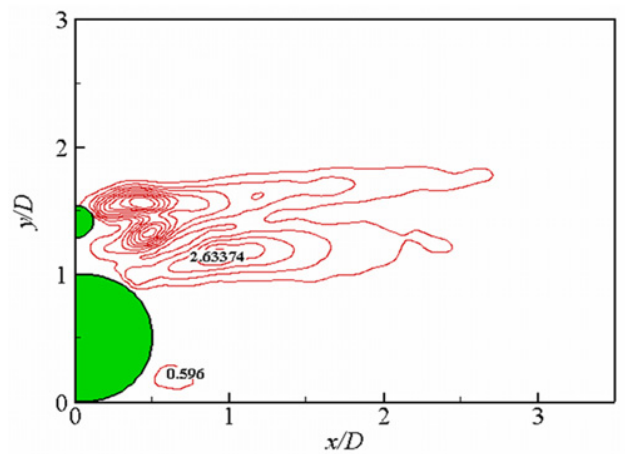

(c)

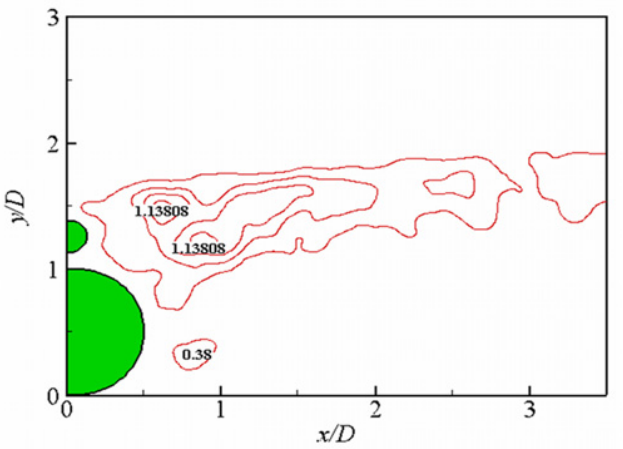

(b)

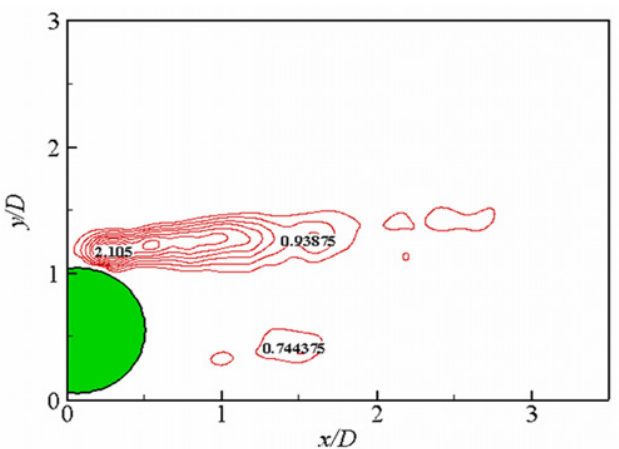

(d)

Fig. 8. Time-averaged fields of the non-dimensional swirling strength for on-bottom piggyback pipelines: $e / D=0, d / D=0.25$, and $R e=3.0 \times 10^{4}$. (a) $G / D=0$, (b) $G / D=0.125$, (c) $G / D=0.30$, and (d) $G / D \approx \infty$ (single pipeline).

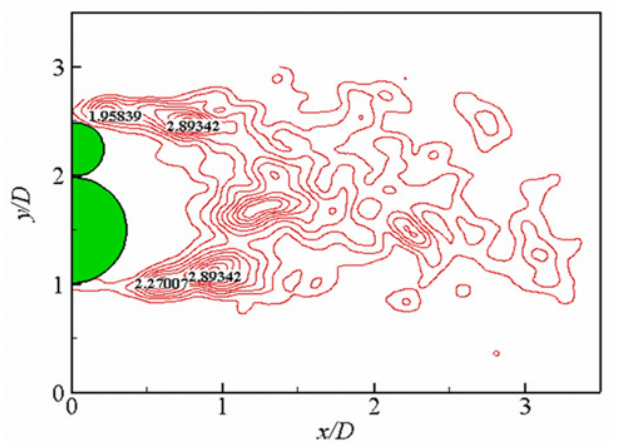

(a)

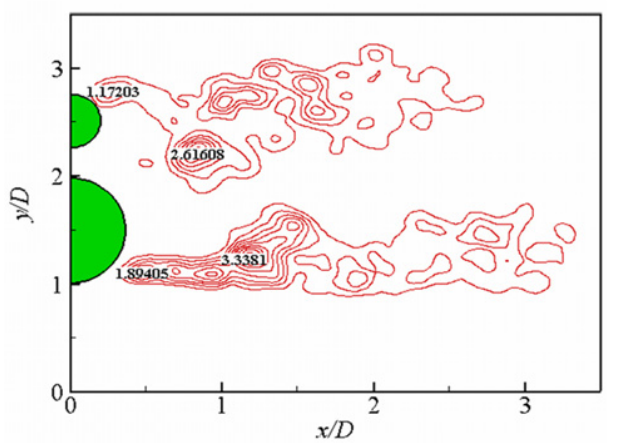

(c)

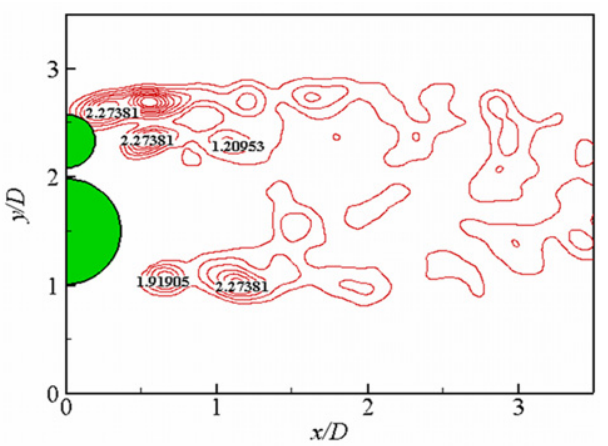

(b)

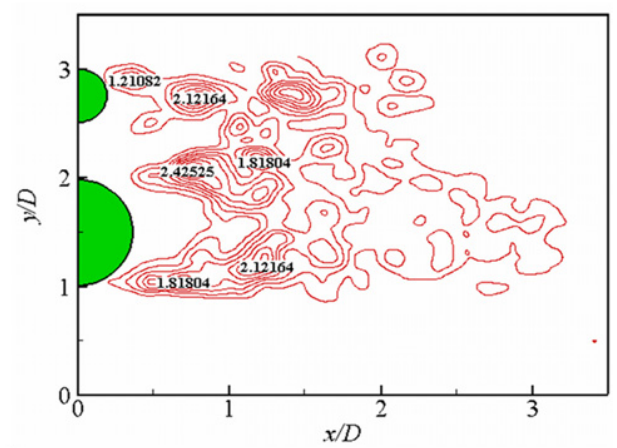

(d)

Fig. 9. Time-averaged fields of the non-dimensional swirling strength for bottom-free piggyback pipelines with a large diameter ratio: (a) $G / D=0$, (b) $G / D=0.10$, (c) $G / D=0.25$, and (d) $G / D=0.50\left(d / D=0.5, e / D=1.0\right.$, and $\left.R e=3.0 \times 10^{4}\right)$. 


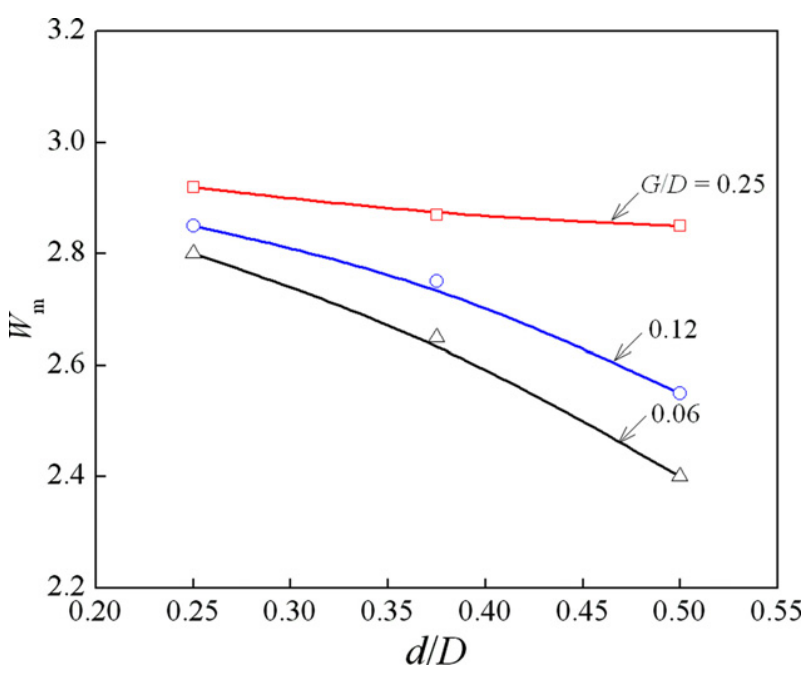

Fig. 10. The maximum non-dimensional swirling strength for piggyback pipelines at various values of $d / D\left(e / D=1.0\right.$ and $\left.R e=3.0 \times 10^{4}\right)$.

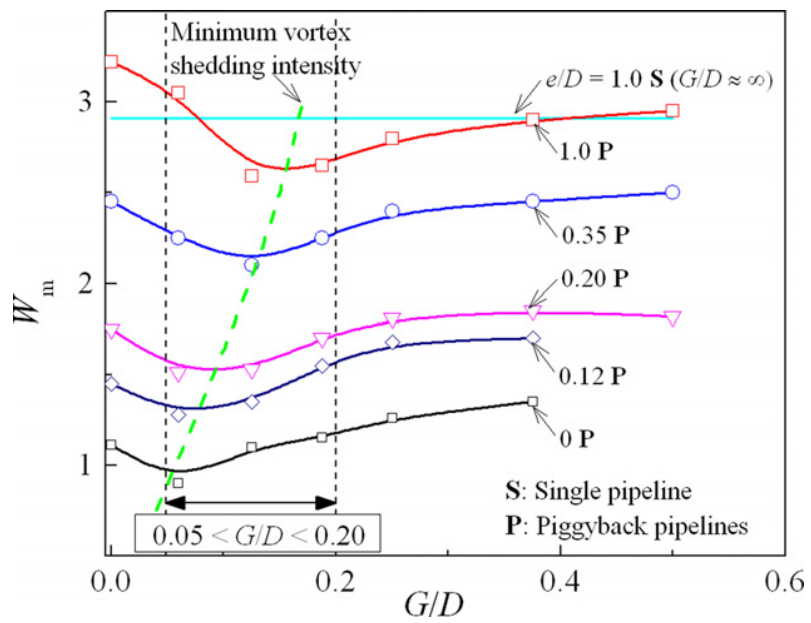

Fig. 11. Variation of the maximum non-dimensional swirling strength $W_{\mathrm{m}}$ with $e / D$ and $G / D\left(d / D=0.25\right.$ and $\left.R e=3.0 \times 10^{4}\right)$.

the trend of $S t$ with $G / D$ is just contrary to that for $W_{\mathrm{m}}$ varying with $G / D$. St number generally increases with the increase of $G / D$ when $G / D$ is at a small value. Then $S t$ decreases with the increase of $G / D$ and reaches a constant value when $G / D>0.3$. It means that the influence of the piggyback on vortex shedding from the main pipe decreases with the increase of $G / D(0.1<G / D<0.3)$ and can be ignored at a large spacing-to-diameter ratio $(G / D>0.3)$. For $e / D=1.0$ (dash dot), St is slightly larger than 0.2 , the value for a wall-free single pipe (dash). The value of $S t$ approaches 0.2 at $G / D=0.5$, owing to the main pipe free from both the piggyback and the bottom. For $e / D=0.2$ (solid line), the largest value of $S t$ occurs at about $G / D=0.06$, which illustrates the vortex shedding from the main pipe was suppressed most significantly at this spacing-to-diameter ratio. For $e / D=0.35$ (dots) and 1.0, the vortex shedding from the main pipe is most significantly inhibited at about $G / D=0.08$ and 0.12 , respectively. This conclusion is coincident with that based on the value of $W_{\mathrm{m}}$.

All the above analyses based on the value of $W_{\mathrm{m}}$ and the vortex shedding frequency $S t$ indicate that the vortex shedding from the main pipe can be suppressed to a certain extent by both the bottom wall and the piggyback. The optimal configuration for the suppression of vortex shedding in Figs. 11 and 12 is G/D ranging in 0.05-0.20.

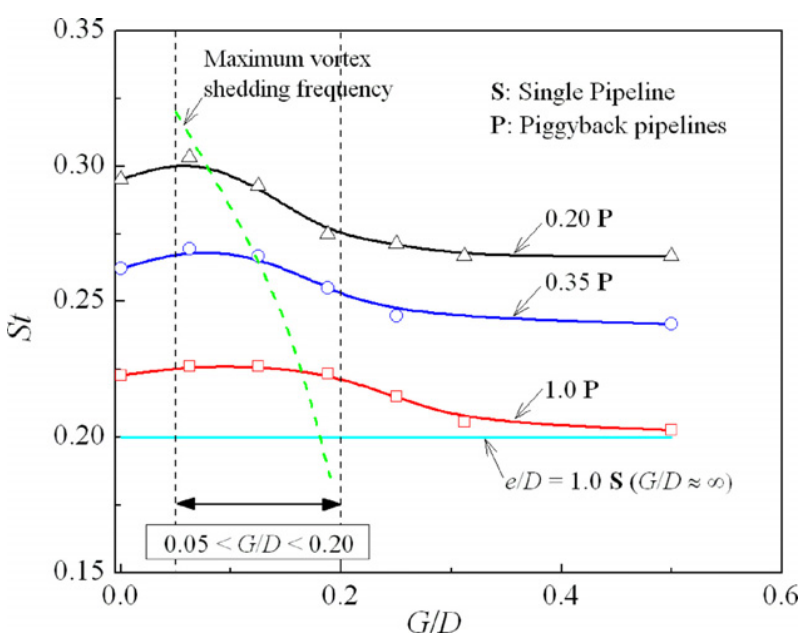

Fig. 12. Variation of $S t$ number for the main pipe with $e / D$ and $G / D(d / D=0.25$ and $R e$ $\left.=3.0 \times 10^{4}\right)$.

\subsubsection{Optimal configurations based on minimum amplitude of VIV}

The amplitudes of VIV for piggyback pipelines were further measured under the same flow condition to verify the optimized configurations obtained from the aspect of vortex shedding suppression. A hydro-elastic facility composed of pulley wheels and springs was used to simulate the VIV of piggyback pipelines and the displacement of the pipeline was measured with a laser sensor. Details of the modeling system of VIV for piggyback pipelines can be referred to in Gao et al. [35].

Although there are many factors determining the characteristics of VIV, including reduced velocity, mass ratio, natural frequency, damping ratio, etc., only $e / D$ and $G / D$ are changed here to study the dynamic response of piggyback pipelines. All the other factors are set constant, with the mass ratio of the piggyback pipelines $m^{*}=1.48$, the natural frequency $f_{\mathrm{n}}=0.78 \mathrm{~Hz}$, and the damping ratio $\zeta=0.067$. The flow condition for VIV measurements is the same as that in Section 3.2, namely, $R e=3.0 \times 10^{4}$. The corresponding reduced velocity is $V_{\mathrm{r}}=$ 6.0 , which is in the $V_{\mathrm{r}}$ range for reaching the maximum amplitude of VIV, according to Det Norske Veritas [36]. The vibrations of piggyback pipelines with different $G / D$ were measured at three gap-to-diameter ratios, i.e. $e / D=0.2,0.35,1.0$. As for the small value of $e / D$, the displacement of VIV is not symmetric about the equilibrium position as is the case for a wall-free condition. The displacement away from the bottom is larger than that toward the bottom due to the pipelines touching the bottom. Hence the displacement away from the bottom will be considered as the maximum amplitude of VIV, denoted as $A$.

The non-dimensional amplitude $A / D$ of VIV for piggyback pipelines varying with $e / D$ and $G / D$ is shown in Fig. 13 and compared with that for a single wall-free pipe (dotted, $A / D=0.8$ ). $A / D$ generally decreases with the decrease of $e / D$ when $G / D$ is set at a constant value, owing to the suppression effect of the bottom wall. For the wall-free piggyback pipelines $(e / D=1.0)$, when the piggyback is contacting the main pipe, the amplitude of VIV is larger than that for a single pipe. This can be explained by the value of swirling strength around the piggyback pipeline with the same configuration as shown in Fig. 11. $A / D$ decreases with the increase of $G / D$ and reaches the smallest value at about $G / D=0.2$. Then $A / D$ increases slightly with the increase of $G / D$ and tends to be at a constant value of that for a single pipe when $G / D$ is at a large value $(G / D>0.3)$. That means the influence of the piggyback would be ignored when the two pipes are free from each other. For $e / D=0.35$, the variation of $A / D$ with $G / D$ is similar to that for $e / D=0.0$. For $e / D=0.2$, the value of $A / D$ is much smaller than that for other gap ratios owing to the significant suppression by the bottom wall. $A / D$ generally increases with the increase of $G / D$ when 


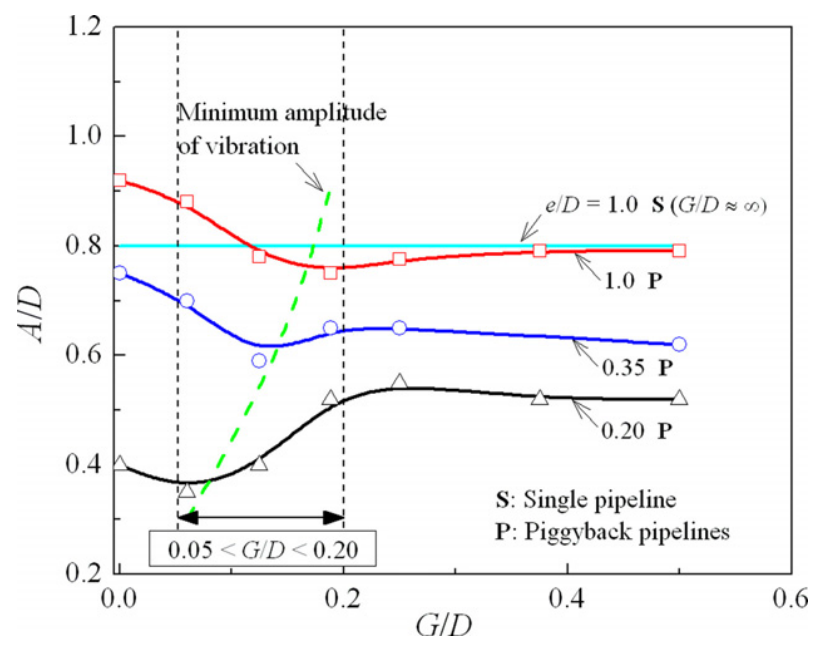

Fig. 13. Amplitudes of VIV for piggyback pipelines with $e / D$ and $G / D\left(m^{*}=1.48, \zeta=\right.$ $0.067, d / D=0.25$, and $V_{r}=6.0$ ).

$G / D<0.2$, then $A / D$ tends to be at a constant value.

Fig. 13 showed that the trend of $A / D$ varying with $e / D$ and $G / D$ is similar to that for $W_{\mathrm{m}}$ (see Fig. 11). It is also indicated that the nondimensional swirling strength $W$ is a key parameter in quantifying the strength of vortex shedding and the suppression of the vortex shedding. The existence of piggybacks has significant influence on the vortex shedding from the main pipe and the amplitude of vibrations. The optimal configuration of piggyback pipelines is for $G / D$ ranging in $0.05-0.20$.

\section{Conclusions}

The vortex shedding characteristics of the near-bed piggyback pipelines in a steady current were studied experimentally. The flow visualizations and quantitative measurements of lee-wake flows around the piggyback pipelines were conducted with a specially arranged PIV system with the upward-illumination of pulsed laser arrays from the bottom of the water flume. The non-dimensional swirling strength was deduced and employed to analyze the vortex shedding intensity of piggyback pipelines. The results of benchmark tests indicated that the vortex shedding characteristics can be well represented by the non-dimensional swirling strength. The effects of configuration factor, including $e / D, G / D$ and $d / D$ on vortex shedding intensity and frequency for piggyback pipelines were investigated. Optimal configurations for piggyback pipelines were also proposed based on the minimum vortex shedding intensity and the corresponding amplitude of VIV. The conclusions from this study can be drawn as below:

(1) Time-averaged swirling strength analyses indicate that the lee-wake patterns for the near-bed piggyback pipelines are dependent on the configuration factors, including the gap-todiameter ratio $(e / D)$, the spacing-to-diameter ratio $(G / D)$, and the diameter ratio of two pipes $(d / D)$, etc. The swirling strength of lee-wake for the piggybacks is obviously asymmetric due to the bed proximity. For a small value of $d / D$, four different vortex shedding modes can be found in the wake regions of the piggyback pipelines dependent on $G / D$ and $e / D$.

(2) For the fixed values of $G / D$ and $d / D$, the maximum swirling strength $\left(W_{\mathrm{m}}\right)$ decreases with the decrease of $e / D$. Moreover for the fixed values of $e / D$ and $d / D, W_{\mathrm{m}}$ decreases with the increase of $G / D$ when $G / D$ is relatively small. Then $W_{\mathrm{m}}$ increases with the increase of $G / D$ and keeps constant when $G / D$ is at a large value $(G / D>0.35)$. The trend of vortex shedding frequency $S t$ varying with $G / D$ and $e / D$ is contrary to that of $W_{\mathrm{m}}$.
(3) For the examined $G / D$ range $(0 \leq G / D \leq 0.5)$, the minimum values of $W_{\mathrm{m}}$ and the corresponding VIV amplitude for the piggyback pipelines emerge within approximately the same range of spacing-to-diameter ratio, i.e. $G / D \approx 0.05-0.20$.

\section{Acknowledgement}

This work is financially supported by National Natural Science Foundation of China (Grant nos. 51109202; 11232012).

\section{References}

[1] Bearman PW, Zdravkovich MM. Flow around a circular cylinder near a plane boundary. Journal of Fluid Mechanics. 1978;89(1):33-47.

[2] Grass AJ, Raven PWJ, Stuart RJ, Bray JA. The influence of boundary layer velocity gradients and bed proximity on vortex shedding from free spanning pipelines. Journal of Energy Resources Technology. 1984;106(1):70-78.

[3] Lei CW, Cheng L, Kavanagh K. Re-examination of the effect of a plane boundary on force and vortex shedding of a circular cylinder. Journal of Wind Engineering and Industrial Aerodynamics. 1999;80:263-286.

[4] Price SJ, Sumner D, Smith JG, Leong K, Païdoussis MP. Flow visualization around a circular cylinder near to a plane wall. Journal of Fluids and Structures. 2002;16(2):175-191.

[5] Wang XK, Tan SK. Near-wake flow characteristics of a circular cylinder close to a wall. Journal of Fluids and Structures. 2008;24(5):605-627.

[6] Lin WJ, Lin C, Hsieh SC, Dey S. Flow characteristics around a circular cylinder placed horizontally above a plane boundary. Journal of Engineering Mechanics. 2009;135(7):697-716.

[7] Li YC, Zhang NC. The hydrodynamic characteristic of submarine piggyback pipeline in wave-current coexisting field. In: Proceedings of fourth international offshore and polar engineering conference (ISOPE), vol. 2. Osaka, Japan; 1994. pp. $10-5$

[8] Chung JS, Conti RJ. Flow-induced torsional moment and vortex suppression for a circular cylinder with cables. In: Proceedings of the fourth international offshore and polar engineering conference, vol. 3. ISOPE, Osaka, Japan; 1994. pp. 447-59

[9] Kalahatgi SG, Sayer PG. Hydrodynamic forces on piggyback pipeline configurations. Journal of Waterway, Port, Coastal and Ocean Engineering. 1997;123(1):16-22.

[10] Williamson CHK. Fluid forces on a small cylinder in the presence of a large cylinder in relative oscillatory flow. Applied Ocean Research. 1985;7(3):124127.

[11] Zeitoun H, Branković M, Tørnes K. Dynamic stability response of piggyback pipelines. In: Proceedings of the 29th international conference on ocean, offshore and arctic engineering (OMAE), vol. 5. Shanghai, China. 2010, pp. 731739 .

[12] Branković M, Zeitoun $H$, Sutherland J. Physical modeling of hydrodynamic loads on piggyback pipelines in combined waves and currents. In: Proceedings of the 29th international conference on ocean, offshore and arctic engineering (OMAE), vol. 5. Shanghai, China. 2010, pp. 933-942.

[13] Kamarudin MH, Thiagarajan KP, Czajko A. Analysis of current-induced forces on offshore pipeline bundles. In: Fifth international conference on computational fluid dynamics in the process industries, vol. 1. Australia. 2006, pp. 1-6.

[14] Kamarudin MH, Thiagarajan KP, Czajko A. Combined effects of waves and currents on offshore pipeline bundles. In: Proceedings of the 26th international conference on ocean, offshore and arctic engineering, vol. 3. San Diego, USA 2007, pp. 337-345.

[15] Zhao M, Cheng L, Teng B, Liang DF. Numerical simulation of viscous flow past two circular cylinders of different diameters. Applied Ocean Research. 2005;27(1):39-55.

[16] Zhao M, Cheng L, Teng B, Dong GH. Hydrodynamic forces on dual cylinders of different diameters in steady currents. Journal of Fluids and Structures. 2007;23(1):59-83.

[17] Zhao M, Cheng L, Teng B. Numerical modeling of flow and hydrodynamic forces around a piggyback pipeline near the seabed. Journal of Waterway, Port, Coastal and Ocean Engineering. 2007;133(4):286-295.

[18] Norberg C. LDV-measurements in the near wake of a circular cylinder. In: Proceedings of the conference on bluff body wakes and vortex-induced vibration. Washington, DC, USA. 1998, pp. 1-12.

[19] Mahir N, Rockwell D. Vortex formation from a forced system of two cylinders. Part I. Tandem arrangement. Journal of Fluids and Structures. 1996;10(5):473489.

[20] Brøns M, Thompson MC, Hourigan K. Dye visualization near a three-dimensional stagnation point: application to the vortex breakdown bubble. Journal of Fluid Mechanics. 2009;622:177-194.

[21] Scarano F, Riethmuller ML. Iterative multigrid approach in PIV image processing with discrete window offset. Experiments in Fluids. 1999;26:513-523.

[22] Schlichting H, Gersten K. Boundary-layer theory. Berlin Heidelberg: SpringerVerlag; 2000.

[23] Lugt HJ. The dilemma of defining a vortex. Recent Developments in Theoretical and Experimental Fluid Mechanics. 1979:309-321. 
[24] Hunt JCR, Wray AA, Moin P. Eddies, stream, and convergence zones in turbulent flows. Center for Turbulence Research Report CTR-S88 1988; 193-208.

[25] Hussian F, Hayakawa M. Eduction of large-scale organized structure in a turbulent plane wake. Journal of Fluid Mechanics. 1987;180:193-229.

[26] Bisset DK, Antonia RA, Browne LWB. Spatial organization of large structures in the turbulent far wake of a cylinder. Journal of Fluid Mechanics. 1990;218:439461.

[27] Adrian RJ, Christensen KT, Liu ZC. Analysis and interpretation of instantaneous turbulent velocity fields. Experiments in Fluids. 2000;29(4):275-290.

[28] Chong MS, Perry AE, Cantwell BJ. A general classification of three-dimensional flow field. Physics of Fluids. 1990;A(2):765-777.

[29] Vollmers H. Detection of vortices and quantitative evaluation of their main parameters from experimental velocity data. Measurement Science and Technology. 2001;12(8):1199-1207.
[30] Zhou J, Adrian RJ, Balachandar S, Kendal TM. Mechanisms for generating coherent packets of haripin vortices in channel flow. Journal of Fluid Mechanics. 1999;387:353-359.

[31] Product-manual for Davis 7.2 software. LaVisionGmbh, Göttingen, Germany; 2009

[32] Ma X, Karamanos GS, Karniadakis GE. Dynamics and low-dimensionality of a turbulent near wake. Journal of Fluid Mechanics. 2000;410:29-65.

[33] Govardhan R. Williamson CHKMean and fluctuating velocity fields in the wake of a freely vibrating cylinder. Journal of Fluids and Structures. 2001;15:489-501.

[34] Sumer BM, Fredsøe J. Hydrodynamics around cylindrical structures. Singapore: World Scientific; 1997.

[35] Gao FP, Yang B, Wu YX, Yan SM. Steady current induced seabed scour around a vibrating pipeline. Applied Ocean Research. 2006;28:291-298.

[36] Det Norske Veritas. Free spanning pipelines. Recommended practice DNV-RPF105; 2006. 